\title{
Heterogeneous Multi-Dimensional Integrated Circuit for Internet-of-Things Application
}

\author{
Nazek El-Atab \\ mmh Labs, Computer, \\ Elecctrical and \\ Mathematical Sciences \\ and Engineering Division \\ King Abdullah University \\ of Science and \\ Technology \\ Thuwal, Saudi Arabia \\ nazek.elatab@kaust.edu.s
} a

\author{
Sohail F. Shaikh \\ mmh Labs, Computer, \\ Elecctrical and \\ Mathematical Sciences \\ and Engineering Division \\ King Abdullah University \\ of Science and \\ Technology \\ Thuwal, Saudi Arabia \\ sohailfaizan.shaikh@kaus \\ t.edu.sa
}

\author{
Sherjeel Khan \\ mmh Labs, Computer, \\ Elecctrical and \\ Mathematical Sciences \\ and Engineering Division \\ King Abdullah University \\ of Science and \\ Technology \\ Thuwal, Saudi Arabia \\ sherjeel.khan@kaust.edu. \\ $\underline{\text { sa }}$
}

\author{
Joho Yun \\ mmh Labs, Computer, \\ Elecctrical and \\ Mathematical Sciences \\ and Engineering Division \\ King Abdullah University \\ of Science and \\ Technology \\ Thuwal, Saudi Arabia \\ joho.yun@kaust.edu.sa
}

\author{
Muhammad Mustafa \\ Hussain \\ mmh Labs, Computer, \\ Elecctrical and \\ Mathematical Sciences \\ and Engineering Division \\ King Abdullah University \\ of Science and \\ Technology \\ Thuwal, Saudi Arabia \\ Elecctrical Engineering \\ and Computer Science \\ University of California, \\ Berkeley \\ California, USA \\ muhammad.hussain@kau \\ st.edu.sa \\ mmhussain@berkeley.edu
}

\begin{abstract}
A novel CMOS-enabled heterogeneous integration and packaging technology based on multi-dimensional (MD) integration of thin-film components to combine between the best of SoC IC integration and best of SiP package integration is demonstrated. As a proof-of-concept, we demonstrate a prototype of a cubic MD-IC (4D system) that includes multifunctional sensors, antenna, microcontroller, light-emitting-diode (LED), a micro-lithium-ion battery and a solar cell. Devices are fabricated on both sides of each substrate ( $\mathrm{Si}, \mathrm{Ge}$ and $\mathrm{GaSb})$ and are interconnected using through-silicon-vias (TSVs) and side interlocks. PDMS encapsulation shows improved mechanical performance. The resulting system is a complete, multifunctional, lightweight, high-performance and compact packaged system. The MD-IC package passed preliminary component-level and system-level reliability tests. Finally, the MD-IC provides an enhanced yield and cost of the system due to the capability to fabricate and test smaller dies separately before integrating them into a complete package.
\end{abstract}

Keywords-3D integration, packaging, heterogeneous, TSVs, reliability test

\section{INTRODUCTION}

Driven by the growing demand for Internet of Thing (IoT) devices, existing integrated circuits (IC) packaging is being pushed beyond its technology limitation to meet new requirements such as the integration of high-performance silicon microprocessors (SoC) with other technologies, such as sensors, high bandwidth memory, high performance RF and communications and high efficiency energy harvesting devices for instance [1-4]. Concurrently, there is a demand for a higher density of active components to reduce the physical size of the heterogeneous electronics package. This work describes a novel approach to meeting both of these demands using CMOS

KAUST Office of Sponsored Research OSR-2015-Sensors-2707, KAUST-KFUPM special initiative OSR-2016-KKI-2880. technology enabled MD-IC - the state-of-the-art silicon technology - that exploits using both sides of silicon wafers combined with joining them to other wafers in a 3D puzzle-like manner. The other wafers need not be $\mathrm{Si}$ as demonstrated by this work where Ge and GaSb wafer are used.

\section{SYSTEM FABRICATION}

The integration and packaging of the MD-IC is completed through a CMOS compatible approach based on $\mathrm{CO}_{2}$ laser patterning and DRIE Bosch process etching of a single-side polished Si wafer (Fig. 1). Each side of the MD-IC has a number of interlocks to connect adjacent sides in a puzzle-like fashion. In addition, TSVs connect the top and bottom sides of each substrate. The number of interlocks and vias, size of the MD-IC and its shape can be customized according to the

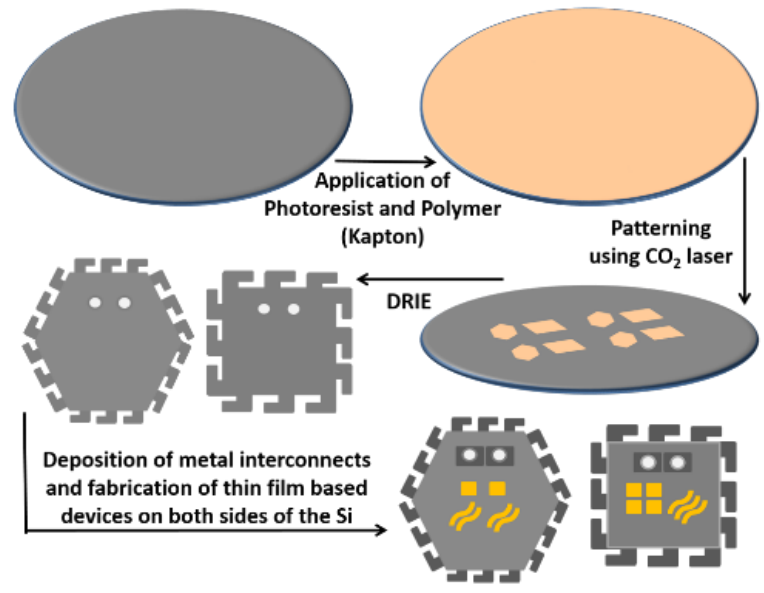

Fig. 1. Schematic of the integration process of the MD-IC 


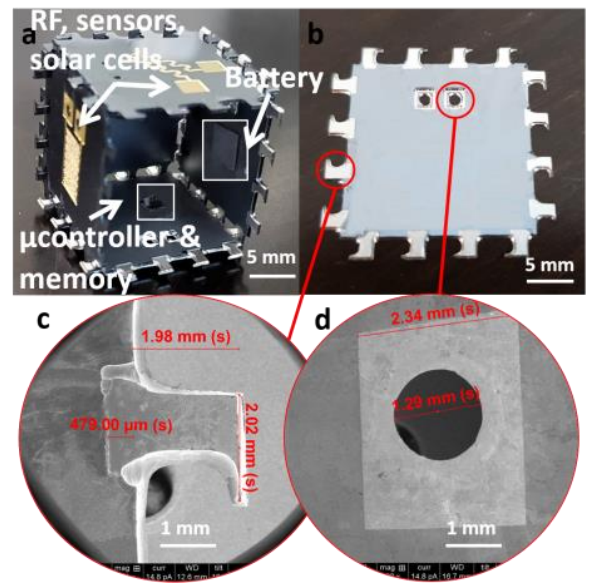

Fig. 2. (a) Inner area of the MD-IC (b) Back-side in the MD-IC showing the metal coated interlocks and TSVs. c) SEM image of the interlock. d) SEM image of the TSV.

application requirements (Figs. 2a-b). Electronic components that do not require $\mathrm{Si}$ as an active material can be fabricated on the backside of a single-side polished $\mathrm{Si}$ wafer, whereas transistors, memory devices and solar cells can be fabricated on the polished front-side of the wafer which has a low density of defects. Humidity/temperature/pressure/pH level sensors and copper ring antenna are fabricated on the outer sides of the MD-IC while a microcontroller IC $(16 \mathrm{MHz})$ and solid-state micro-lithium-ion battery are embedded on the inner sides (Fig. 2a). Indium gallium zinc oxide (IGZO) thin film transistors (TFT) are also fabricated on the Si faces for sensing application. The interconnects are coated with 500-nm silver using e-beam evaporation (Figs. 2b-d). The same process has been applied on $\mathrm{Ge}$ and $\mathrm{GaSb}$ wafers to show that heterogeneous materials can be integrated in the MD-IC for an optimized performance of the system. Six layers of PDMS are used to encapsulate the interconnects and isolate the inner components from outer environment and enhance the mechanical robustness of the system (Fig. 3) [5].

\section{RESULTS AND DISCUSSION}

Electrical characterization on the system is conducted to test the reliability of side interlocks and TSVs in interconnecting the different sides and faces of the cubic system. Results show that opposite faces of the same side are interconnected though a $0.2 \Omega \mathrm{TSV}$, while adjacent sides are

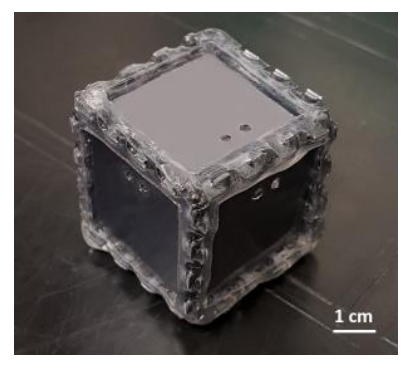

Fig. 3. PDMS encapsulated MD-IC for enhanced mechanical robustness.
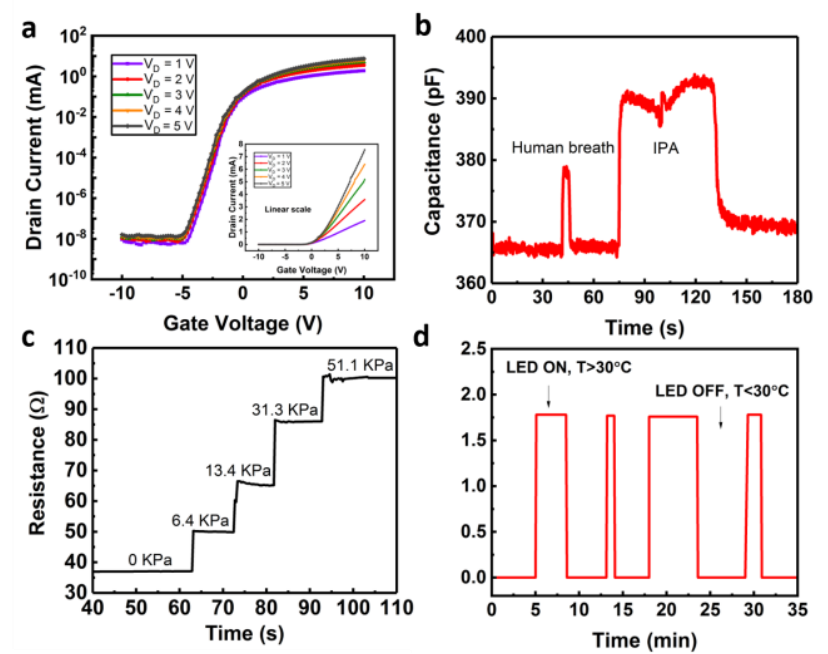

Fig. 4. (a) Transfer characteristic $\mathrm{I}_{\mathrm{D}}-\mathrm{V}_{\mathrm{G}}$ of the IGZO-based TFT in linear and logarithmic scales, $\mathrm{L}=10 \mu \mathrm{m}$ and $\mathrm{W}=200 \mu \mathrm{m}$ (b) Graphene-based humidity sensor output when exposed to human breath and IPA. (c) Graphene-basec pressure sensor output. (d) Current output from the microcontoller when the outer temperature MD-IC is increased above a threshold $30^{\circ} \mathrm{C}$.

interconnected via $11.1 \Omega$ side interlocks. The TFT in addition to the humidity and pressure sensors are characterized when exposed to different stimuli (Fig. 4a-c). A system-level reliability test is conducted on the MD-IC - as a standalone system - by programming the microcontroller such that an LED turns on when the temperature of the MD-IC system rises above a threshold value of $30^{\circ} \mathrm{C}$ (Fig. $4 \mathrm{~d}$ ). In this test, all of the devices are fabricated on different faces and sides of the MDIC. The MD-IC passed component-level reliability tests including un-biased highly accelerated stress test ( $\mu$ HAST) $200^{\circ} \mathrm{C}$ and $300^{\circ} \mathrm{C}$ at $60 \% \mathrm{RH}$ humidity for 48 hours and environmental test (immersion in de-ionized and saline waters) 48 hours. The current output from the microcontroller within the MD-IC during standby mode is similar to reference values. This confirms the CMOS integration capability on the different faces of the MD-IC.

\section{CONCLUSION}

Integrated electron device manufacturers are persistently being confronted with device integration challenges as consumers desire electronics to be of smaller size, cheaper and more multi-functional than ever. In this work, we introduced the concept of multi-dimensional integrated circuits (MD-IC) which combines between the best of the existing integration and packaging technologies and which promises high performance, low power consumption and multi-function features within one small form factor package. Reliability qualifications showed that the MD-IC system provides excellent and stable results even under extreme conditions which makes it promising for harsh environment applications.

\section{REFERENCES}

[1] R. Aitken, VLSI, 2014, pp. 1-2. [2] S. Pendharkar, VLSI, 2016, pp. 1-2 [3] C. H. Douglas, IEDM, 2014, pp. 27. [4] R. Tummala, IEEE Transactions on Advanced Packaging, 27, 241, 2014. [5] N. El-Atab, S.F. Shaikh, S.M. Khan, and M.M. Hussain, Adv. Eng. Mat., p. 1900043, 2019. 